1 Secretaria Municipal de Saúde de Florianópolis Florianópolis (SC), Brasil. Orcid: https://orcid. org/0000-0001-6663$352 X$

lucas.enfermagem@gmail. com

2 Secretaria Municipal de Saúde de Palhoça Palhoça (SC), Brasil. Orcid: https://orcid. org/0000-0002-74252660

daya_rocha90@hotmail.com

3 Universidade Federal de Santa Catarina (UFSC) -

Florianópolis (SC), Brasil. Orcid: https://orcid. org/0000-0001-60341497

yanaatomasi@gmail.com

\section{A vigilância do território na atenção primária: contribuição do agente comunitário na continuidade do cuidado}

\author{
Surveillance of the territory in health in primary care: the \\ contribution of the Community Health Worker in the continuity of \\ care
}

Lucas Alexandre Pedebos', Dayana Karla Rocha², Yaná Tomasi3

DOI: $10.1590 / 0103-1104201811912$

RESUMO O presente estudo teve como objetivo identificar fragilidades no processo de trabalho dos Agentes Comunitários de Saúde (ACS) e a percepção de importância dada por eles sobre as ações em vigilância do território para o trabalho da equipe de saúde, juntamente a um processo de sensibilização e capacitação dos ACS sobre a temática. Para tal, foi realizado cruzamento de dados de dois sistemas de informação (prontuário eletrônico e cadastro das famílias) e aplicação de questionário aos ACS, com posterior processo educativo junto a esses atores sobre a temática, a partir das fragilidades identificadas. Os resultados demonstram que os ACS apresentam consciência da importância do seu trabalho sobre o tema estudado, mas que as atribuições específicas da categoria não eram desempenhadas em sua plenitude, observação feita, principalmente, no cruzamento dos dados. O processo de sensibilização parece tê-los ajudado na identificação de fragilidades no processo de trabalho. Percebe-se, assim, que a vigilância do território parece estar presente no discurso dos ACS, mas a prática cotidiana evidência fragilidades no seu processo de trabalho e na comunicação com o restante da equipe, agravadas pela existência de sistemas de informação desconexos.

PALAVRAS-CHAVE Vigilância em saúde pública. Estratégia Saúde da Família. Agente Comunitário de Saúde.

ABSTRACT This study aimed to identify weaknesses in the work process of the Community Health Workers $(\mathrm{CHW})$ and their perception of importance given by them to the actions in territorial surveillance for the work of the health team, together with a process of awareness and training of the $\mathrm{CHW}$ on the subject. For this purpose, a data cross-referencing of two information systems (electronic medical record and family register) was carried out and a questionnaire was applied to the $\mathrm{CHW}$, with subsequent educational process with these actors on the theme, based on the fragilities identified. The results show that the CHW are aware of the importance of their work on the subject studied, but that the specific assignments of the category were not fulfilled in their fullness, an observation mainly done in the crossreferencing data. The sensitization process seems to have helped them to identify weaknesses in their work process. It is thus perceived that the territorial surveillance seems to be present in the discourse of the $\mathrm{CHW}$, but the daily practice shows weaknesses in their work process and communication with the rest of the team, aggravated by the existence of disconnected information systems.

KEYWORDS Public health surveillance. Family Health Strategy. Community Health Worker. 


\section{Introdução}

Em 1987, no Ceará, ocorreu a primeira experiência de Agente Comunitário de Saúde (ACS) de forma estruturada, a fim de criar oportunidade de emprego para as mulheres da área da seca, bem como reduzir os níveis de mortalidade infantil. Oficializado como Programa de Agentes Comunitários em Saúde (Pacs), em 1991, também no Ceará, seria incorporado, posteriormente, ao Programa Saúde da Família (PSF), em 1994, colocando o ACS como parte de uma equipe multiprofisisonal'.

Porém, a relação entre esses dois programas - Pacs e PSF/Estratégia Saúde da Família (ESF) - não foi estabelecida inicialmente, de modo que por algum tempo funcionaram como programas paralelos ${ }^{2}$ O trabalhado que o ACS já executava foi parcialmente incorporado pelos demais membros da equipe, sendo incumbidas a esse profissional outras atribuições que as equipes de saúde supunham ser importantes. As atribuições a partir dessa incorporação só seriam estabelecidas na Política Nacional de Atenção Básica (PNAB), em 2006.

No ano de 2011, foi publicada a Portaria ${ }^{\circ}$ 2.488/11, alçando o PSF à ESF como iniciativa para a reorganização do modelo de distribuição e inter-relação dos serviços de saúde do País e colocando a ESF como modelo prioritário de organização da Atenção Primária à Saúde (APS) ${ }^{3}$. Por meio da ESF, buscou-se a expansão, a qualificação e a consolidação da APS com a reorientação do processo de trabalho para essa estratégia, com maior potencial de aprofundar os princípios, as diretrizes e os fundamentos de um sistema universal, bem como ampliar a resolutividade e o impacto na situação de saúde das pessoas e coletividades através de suas equipes.

Por meio da atuação das Equipes de Saúde da Família (EqSF), de forma multiprofissional, o ACS tem função fundamental na medida em que realiza ações de prevenção de doenças e promoção de saúde por meio de ações educativas individuais e coletivas ${ }^{2}$. Ao analisar as potencialidades do ACS na APS, percebe-se ser ele um elo importante entre a equipe e a comunidade, por poder contribuir para o acompanhamento das proposições de tratamento devido à forma facilitada com que transita no cotidiano sociofamiliar das pessoas ${ }^{4-6}$.

A APS, em muitos países, utiliza um modelo de adscrição populacional baseado em listas fechadas, estando as pessoas diretamente vinculadas às equipes de saúde - com liberdade de escolha da pessoa, normalmente ${ }^{7}$. No entanto, devido à importante situação de fragilidade social em que se encontra boa parte da população brasileira e à situação íntima que o processo saúde-doença possui com a sua dinâmica social, o modelo nacional optou por adscrever as equipes a um território. Assim, as equipes se tornam responsáveis pelas pessoas e suas famílias, ao mesmo tempo que conhecem as fragilidades e potencialidades do meio em que estão inseridas 4 .

Essa forma de organização da APS possui consonância no modelo de vigilância em saúde, a qual busca ampliar a concepção de saúde ao considerá-la resultante das condições de vida, não somente como ausência de doença, tendo como objeto de atenção a compreensão do sujeito e seu contexto de vida, passando pela garantia de ações integrais que visam a prevenir doenças e promover a saúde. Assim, torna-se imprescindível uma abordagem diferenciada dessa forma de intervenção, e cabe à ESF a integração e a organização das atividades em seu território adscrito, a fim de prestar uma atenção contínua e integral que possibilite $o$ desenvolvimento de ações capazes de responder aos reais problemas da população $0^{\mathbf{4}, 8}$.

No município de Florianópolis (SC), os ACS utilizam como ferramenta de registro de trabalho o Sistema de Cadastro de Família (CadFam), desenvolvido pela Secretaria Municipal de Saúde com o objetivo de agregar informações sobre o cadastramento familiar e as visitas às famílias. Ainda, também desenvolvido por essa instituição, existe o programa InfoSaúde, que funciona como prontuário eletrônico, sendo alimentado pelos profissionais de nível superior e técnicos de enfermagem atuantes na rede de saúde municipal. Entretanto, cabe ressaltar 
que esses dois Sistemas de Informação em Saúde (SIS) não são interligados e que são preenchidos por grupos diferentes de profissionais, facilitando a ocorrência de discrepâncias de dados entre ambos.

A observação de diferenças nos registros desses dois sistemas, encontradas no cotidiano da prática assistencial dos pesquisadores, levou ao questionamento dos motivos pelos quais esses problemas estariam acontecendo. Tais observações foram feitas em reuniões da EqSF a partir do acompanhamento de indivíduos (pacientes) específicos, mas, devido à recorrência dessas divergências, os pesquisadores formularam a hipótese de que esse problema poderia ser sistemático, principalmente ao saberem que os dois sistemas (CadFam e InfoSaúde) não possuem integração para compartilhamento dos dados. Dessa forma, os registros feitos pelo ACS para o indivíduo ‘João da Silva' no CadFam não atualizam os registros para o mesmo indivíduo no InfoSaúde, levando ao risco de que ações realizadas pelo ACS não sejam percebidas pelo restante da equipe ao analisar o prontuário; ou de que as ações registradas no prontuário pelo médico ou enfermeiro não sejam direcionadoras das ações do ACS no território, na comunidade e no domicílio para o mesmo indivíduo. Assim, fica restrita à reunião de equipe o único momento de compartilhamento dessas informações, a qual pode não ser suficiente para a discussão de todos os casos devido ao elevado volume de trabalho.

No entanto, a desintegração dos sistemas seria suficiente para explicar essas diferenças, já que muitas divergências aconteciam entre pacientes de acompanhamento próximo e continuado, como portadores de doenças crônicas, gestantes e crianças? Ou haveria algum fator intrínseco ao trabalho dos ACS, como o não entendimento da importância desses elementos para a promoção de saúde na população? A vigilância do território estaria devidamente integrada ao processo de trabalho do ACS, sobretudo nos pacientes que mais exigem continuidade do cuidado? Tais questionamentos foram os principais motivadores que levaram os pesquisadores a escolherem o tema do trabalho, o qual possui como objetivos a identificação dos principais elementos que levam a essas fragilidades no trabalho do ACS relativos à vigilância do território e à continuidade do cuidado, bem como à realização de atividades educativas com esse público que visassem à minimização dos problemas identificados. A proposição de melhorias do cenário assistencial, além de parte do compromisso do programa de residência multiprofissional no qual os pesquisadores estão inseridos, compõe um elemento do método escolhido para este trabalho.

\section{Métodos}

O presente trabalho foi desenvolvido em uma Unidade Básica de Saúde (UBS) componente da rede de Atenção Básica da Secretaria Municipal de Saúde de Florianópolis (SC). Essa UBS dispõe da atuação de 2 EqSF, sendo a pesquisa desenvolvida na equipe de saúde que conta com a atuação de residentes vinculados à Residência de Medicina de Família e Comunidade, da Prefeitura Municipal de Florianópolis (PMF), e à Residência Multiprofissional em Saúde da Família, da PMF e da Universidade do Estado de Santa Catarina (Udesc), sendo dois dos três pesquisadores integrantes de um desses programas.

Para alcançar os objetivos propostos e devido à inserção dos autores do trabalho na prática assistencial, optou-se pela Pesquisa do tipo Convergente Assistencial (PCA), que requer a participação ativa dos participantes e dos pesquisadores, sendo orientada para a resolução ou minimização de problemas na prática, realização de mudanças e/ou introdução de novas práticas de saúde?. Entendese que esse método seja adequado tanto pela inserção dos pesquisadores diretamente na prática assistencial em saúde quanto pela identificação prévia de problemas que impactam negativamente na qualidade da assistência ao cidadão, emergidos a partir do relato dos profissionais das EqSF. 
A PCA possui 5 fases distintas que contemplam desde a escolha do tema, a partir da observação da prática, até a síntese do processo em resultados. Elas foram definidas como: concepção (escolha do tema, objetivo de pesquisa e revisão de literatura), instrumentação (definição dos procedimentos metodológicos), perscrutação (coleta de dados pelas técnicas escolhidas), análise (leitura e apropriação dos dados coletados) e interpretação (geração de informação a partir da fase anterior e síntese) ${ }^{9}$. Todas essas etapas foram seguidas, ainda que, para efeito de fluidez do relato, tenha-se optado por não descrever individualmente as atividades de cada um desses momentos.

Os participantes da pesquisa foram os ACS (em número de quatro) atuantes na referida equipe pesquisada, os quais demonstraram aceitar participar desta pesquisa através da assinatura do Termo de Consentimento Livre e Esclarecido (TCLE), após devida explicação dos objetivos, benefícios, potenciais riscos, direito à desistência e segurança das informações coletadas. O presente estudo foi aprovado pelo Comitê de Ética e Pesquisa em Seres Humanos da Udesc, através do parecer $\mathrm{n}^{\mathrm{o}} 1.730 .126$, e seguiu fielmente os preceitos da Resolução no 466/2012.

A coleta de dados ocorreu de duas formas: 1) busca de registros unitária não identificada de pessoas de determinados grupos populacionais (hipertensos, diabéticos, gestantes, crianças menores de 5 anos) nos sistemas CadFam e InfoSaúde referentes ao ano de 2016; 2) aplicação de formulário contendo questões sobre vigilância do território aos participantes da pesquisa, contendo seis questões, quais sejam: 1. Você faz vigilância do território? Se sim, cite exemplos; 2 . Para você, qual a importância da vigilância do território?; 3. Cite fatores que facilitam o seu trabalho de vigilância do território; 4. Cite fatores que dificultam o seu trabalho de vigilância do território; 5 . A Enfermeira da sua área pede para que você faça uma visita a um paciente de sua microárea, hipertenso, e que vem apresentando nas consultas dificuldade para aderir ao tratamento.
O que você faria nesse caso?; 6. O que você orientaria e observaria em uma visita de rotina a uma criança menor de 1 ano de idade? Esse mesmo questionário foi aplicado antes e após o processo de sensibilização e capacitação dos ACS sobre a temática. Adicionalmente, alguns questionamentos sobre os dados encontrados no cruzamento dos sistemas foram feitos aos ACS no momento de sensibilização e capacitação. Essa etapa ocorreu de agosto a dezembro de 2016.

O processo de capacitação emergiu como proposta dos profissionais da equipe, incluindo os ACS, em discussão com os pesquisadores como ferramenta para melhor uso dos dados levantados. Além disso, sugeriu-se, também, a comparação das listagens de pacientes nos dois sistemas, com verificação caso-a-caso junto aos profissionais da equipe para melhor entendimento de onde estariam os problemas.

A análise dos dados ocorreu de duas maneiras distintas: 1) os dados coletados através do CadFam e do InfoSaúde foram cruzados utilizando o programa Microsoft Excel 2007 e métodos adicionais de linkage, para verificar as similaridades das listas de pessoas registradas pelos ACS com aquelas atendidas pela EqSF; 2) as respostas dos formulários foram avaliadas a partir da comparação das respostas nos dois momentos de aplicação, somando-se às anotações dos relatos dos participantes durante o momento de sensibilização e capacitação. Tal etapa ocorreu entre os meses de outubro de 2016 e janeiro de 2017.

\section{Resultados e discussão}

Os números encontrados na comparação entre os Sistemas CadFam e InfoSaúde (tabela 1) demostram diferenças importantes entre os registros dos grupos populacionais selecionados quando comparados os registos dos ACS (CadFam) com os dos demais membros da equipe (InfoSaúde - atendidos pela EqSF). No caso das crianças, cadastradas em maior número no CadFam, é possível que não estejam 
sendo retiradas deste sistema quando completam 5 anos de idade (função não realizada automaticamente pelo sistema, mesmo que a idade tenha ultrapassado os 5 anos) ou que apenas 79 crianças estejam sendo acompanhadas pela EqSF. Quanto às gestantes, apenas 9 estariam em acompanhamento pela EqSF, mas é importante verificar se o cadastro no
CadFam está sendo mantido mesmo após o nascimento do bebê ou se apenas 19 gestantes estavam realizando pré-natal. Entretanto, os ACS afirmaram durante a atividade educativa que conhecem as gestantes das suas microáreas e que estão sempre atentos em reforçar as datas das consultas de pré-natal.

Tabela 1. Número de pessoas em determinados grupos populacionais presentes nos sistemas InfoSaúde e CadFam na UBS em estudo, Florianópolis (SC), 2016

\begin{tabular}{lrrr}
\hline & InfoSaúde & CadFam & Ambos (intersecção entre InfoSaúde e CadFam) \\
\hline Crianças & 136 & 216 & 79 \\
Gestantes & 19 & 61 & 10 \\
HAS e DM & 295 & 158 & 44 \\
\hline
\end{tabular}

Fonte: Elaboração própria.

Entre os portadores de hipertensão e diabetes, também há importante diferença, havendo grande número de pessoas atendidas pela EqSF que não são devidamente acompanhadas no domicílio. As pessoas existentes no CadFam que não aparecem no InfoSaúde podem tanto ser portadoras das referidas enfermidades que não realizam acompanhamento na UBS (usuários do sistema privado, por exemplo) quanto pessoas que mudaram seus endereços, mas não foram descadastradas do território. No entanto, a justificativa dada pelos ACS para as 251 pessoas que acompanham com a EqSF, mas não estão registradas no CadFam, foi vaga, normalmente semelhante a 'todos os hipertensos e diabéticos são acompanhados'.

A gente acompanha todo mundo da nossa área.

[...] Tudo é passado na reunião de equipe pra [cita o nome da enfermeira da equipe] e pro [cita o nome do médico da equipe]. (ACS 1).

Na aplicação dos formulários junto à sensibilização e à capacitação, os ACS participantes da pesquisa demonstraram reconhecer a importância do seu trabalho de vigilância do território para o planejamento das ações de sua respectiva equipe e, consequentemente, para a comunidade, compreendendo que seu trabalho não se resume a identificar problemas no território, mas, também, orientar a população para a prevenção de doenças. Ainda, apresentaram conhecimento mínimo quanto aos sistemas utilizados (InfoSaúde e CadFam), reconhecendo que mesmo aqueles que não são preenchidos por eles dependem de seu papel na vigilância do território.

$\mathrm{Na}$ análise dos formulários aplicados antes da atividade educativa, é identificável a percepção dos ACS sobre a temática quando descreveram, por exemplo, suas condutas nas visitas domiciliares às crianças menores de cinco anos, com a ideia de observar se as vacinas estão sendo feitas e se os pais estão levando os filhos às consultas periódicas.

"Ver vacinas se estão em dia, peso, se está sendo amamentada, consultas mensais em dia, pediatra, enfermeira ou médico" (ACS 3).

Entretanto, em suas falas, não demonstraram lembrar-se das orientações quanto à saúde 
bucal, nem mesmo da importância da amamentação para o desenvolvimento orofacial e da higiene bucal dos bebês. Mesmo as orientações sobre a higiene de prótese em adultos foram falhas, ainda que, nos anos de 2015-2016 todos os profissionais da UBS estudada tenham participado de atividades de educação continuada sobre saúde bucal, inclusive cuidados para prevenção do câncer bucal, dadas as características epidemiológicas da comunidade local. Aliás, como constataram Santos et al. $^{10} \mathrm{em}$ sua pesquisa, a saúde bucal parece não ser um tema para o qual normalmente os ACS recebem capacitação, sendo reservadas apenas orientações restritas para gestantes e bebês. Isso deixa clara a importância da interação de toda a equipe e a realização de atividades de educação permanente para alcançar, da melhor forma possível, atividades de impacto na educação em saúde e vigilância do território.

Os ACS demonstraram, durante a atividade educativa e no preenchimento do formulário (nos dois momentos), que sabem orientar as gestantes quanto aos cuidados básicos durante o pré-natal, acerca dos sinais de alerta na gestação, reforçando a importância do acompanhamento pré-natal. A partir das discussões com a equipe sobre essa temática, formulou-se a hipótese de que as diferenças encontradas nos números de gestantes entre os dois pode se dar pela não remoção do cadastro de gestantes pelos ACS no CadFam, e/ou por haver gestantes que realmente não procuram a UBS; e, se realmente as gestantes não estão comparecendo às consultas, cabe também aos ACS junto à EqSF realizar busca ativa dessas mulheres para a prestação de uma assistência pré-natal adequada.

A gente fala da bolsa [amniótica], das contrações. Até porque aqui todo mundo é mãe, então sabe bem como é, também, além do que a gente aprendeu nos cursos. (ACS 4).

Os ACS citaram que, quando conseguem construir uma relação de vínculo com a comunidade, podem adquirir mais informações e orientar melhor os indivíduos, permitindo que até mesmo os casos mais complexos possam ser relatados a eles e conduzidos à EqSF. Entretanto, adentrar a intimidade de uma família não é tarefa fácil, devendo ser feito com cuidado, buscando respeitar seu formato, suas crenças e seus anseios; e perceber o contexto geral de cada família, habilidade que permite ampliar o olhar para as estratégias a serem adotadas para abordagem das necessidades de cada família, parte integrante da vigilância do território. Ainda que deva agir como facilitador de acesso para os casos nos quais forem identificados critérios prioritários, há de se tomar cuidado para que o ACS não seja visto pela comunidade como alguém que 'barganha' vagas - situação comum, por ser o ACS normalmente morador da mesma comunidade -, o que é minimizado quando a equipe consegue ofertar acesso facilitado ${ }^{11-13}$.

Desde que começou [o acolhimento], ficou mais fácil, porque quando era marcado, antes, as pessoas não conseguiam consulta e, daí, ficaram pedindo pra gente. Agora a gente só marca com a [cita o nome da enfermeira] quando é caso grave [referindo-se a casos sem acompanhamento, não necessariamente com gravidade]. O resto, as pessoas conseguem vir e ser atendidas. (ACS 3).

Quando questionados sobre o reconhecimento da importância da visita domiciliar, percebe-se a compreensão do seu valor, tanto para a coleta de dados quanto para conhecimento da sua localidade; para a equipe, contribui fornecendo informações que auxiliam no planejamento das ações em saúde e no conhecimento das demandas da comunidade; já para os usuários, leva informações do centro de saúde e vice-versa, bem como realiza o trabalho de orientação, promoção e prevenção em saúde.

A partir da análise dos questionários aplicados, observam-se respostas mais elaboradas na primeira etapa. Após o momento de atividade educativa, as respostas foram mais sucintas, mas com o mesmo conteúdo, demonstrando pouca ou nenhuma mudança 
no posicionamento. Apesar disso, os ACS participaram ativamente da sensibilização e da capacitação, mostrando-se engajados nas atividades, expondo suas dúvidas e opiniões; isso deixa claro que os participantes tinham noção do tema abordado e estavam interessados em ser fiéis nas suas respostas, apenas procurando finalizar a atividade o mais brevemente possível.

Na primeira pergunta do questionário Você faz vigilância do território? Se sim, cite exemplos -, optou-se pela lógica dos exemplos para abordar o conhecimento dos participantes quanto à vigilância do território, em vez de perguntar o conceito de vigilância do território, visto que se entendeu como mais fácil para a compreensão dos participantes, sendo que, por consequência, traria respostas mais fidedignas aos pesquisadores. Quando questionados sobre os fatores dificultadores para esse tipo de atividade, os ACS relataram a dificuldade de discutir alguns casos e, por vezes, a demora por parte da equipe para apresentar proposta de abordagem para os casos questionados. Citaram, ainda, que os portadores de Hipertensão Arterial Sistêmica (HAS) e Diabetes Mellitus (DM) também lhes trazem dificuldades, visto que muitos não admitem ter a doença ou preferem não aderir ao tratamento médico. Os ACS informaram que há alguns casos em que os usuários são conhecidamente portadores dessas enfermidades (casos estes discutidos em reunião de equipe, inclusive), mas na visita domiciliar ocorre de negarem a existência de tal problema ou dizerem que já estão 'curados' da doença.

Esse tipo de situação pode sugerir que a abordagem da equipe de saúde, incluindo o ACS, pode não estar sendo adequada ao caso, tanto no aspecto biomédico da doença (no tocante ao seu desenvolvimento, ligado a hábitos de vida, à característica da cronicidade da doença e à importância do tratamento para minimização de problemas em consequência da HAS e DM, alguns destes fatais ou incapacitantes) como em outros aspectos individuais e coletivos vinculados à visão que a pessoa tem sobre o desenvolvimento da doença, ao contexto sociocultural no qual está inserida e mesmo à percepção que se tem sobre doenças assintomáticas - muitas vezes não entendidas como doença pelo usuário ${ }^{14,15}$.

Ainda, a justificativa dada pelos ACS expõe um problema de comunicação importante entre estes e o restante da equipe, já que não deveria haver listagens de usuários diferentes entre eles, o que só acontece por não haver compartilhamento efetivo de informações. A desvinculação dos sistemas de informação certamente é um problema importante nesse processo, já que as ferramentas informatizadas disponíveis não permitem o uso destas como facilitadoras de comunicação, por meio das quais poderia ser feito o direcionamento de condutas e priorização dos casos a serem abordados. Assim, o ACS fica com o trabalho de agente integrador entre a comunidade e o restante da equipe bastante prejudicado ${ }^{16}$.

Por outro lado, quando questionados quanto aos pontos facilitadores, apesar do destaque relacionado à demora da equipe em dar retorno às demandas trazidas por eles, as decisões tomadas lhes são satisfatórias. A colaboração dos moradores se faz de extrema importância para que os ACS possam coletar informações e auxiliar os pacientes, e faz com que a equipe se organize para o atendimento desses indivíduos; de toda forma, a equipe também deve ser acolhedora das demandas dos agentes comunitários, com respostas mais rápidas para os casos transmitidos. Portanto, o trabalho dos ACS junto à equipe e aos moradores pode facilitar o processo de trabalho e trazer vantagens na organização do sistema para atendimento da comunidade, como corroboram os achados de outros artigos $5,16,17$.

Ponto relevante citado pelos ACS durante a atividade educativa foi de que gostariam de contribuir mais para o processo de trabalho, visto que poderiam auxiliar no controle dos hipertensos, já que sabem aferir PA (Pressão Arterial), atividade poucas vezes realizada na frequência orientada pela equipe pela impossibilidade de o hipertenso fazê-lo. Entretanto, 
tal prática não consta como atividade atribuída ao ACS, tanto na Carteira de Serviços de Florianópolis quanto na PNAB ${ }^{2,18}$.

A gente sabe ver pressão. [...] eu acho que é importante porque a gente já está lá na casa da pessoa, daí já mede. Mas eu sei que não pode ficar chacoalhando o aparelho na rua porque estraga ele. (ACS 2).

Ainda, lembraram que, por conta do déficit de servidores técnicos administrativos, os ACS têm realizado atividades como ligação para informar $o$ agendamento de consultas em especialidades e serviços na recepção por meio de escala, mesmo que essas atividades também não constem nos referidos documentos como sendo de sua responsabilidade. Ainda que o fato de o ACS trabalhar dentro da unidade não descaracterize completamente seu papel, uma vez que possa haver conciliação das suas atividades específicas com esse ambiente de saúde, como o registro de novos cadastros e acolhimento inicial ao usuário, diminui-se o tempo disponível para boa parte do processo de vigilância de território, que só pode ser feito na comunidade.

Porém, vale destacar que os dados obtidos nos sistemas de informação foram correspondentes a um ano, o que descarta a possibilidade de ser essa a explicação para a grande diferença encontrada entre os registros dos ACS e o restante da equipe. Não que no período de um ano não seja possível que o ACS realize as visitas domiciliares, preencha o CadFam e ainda atualize o sistema à medida que as mudanças ocorrem no território de atuação, mesmo que com algum atraso, já que os grupos populacionais utilizados no levante para comparação entre os sistemas não estão em situações que mudam rapidamente (gestantes, crianças e doenças crônicas).

Em estudo realizado na cidade de Santa Maria, no Rio Grande do Sul, observa-se que a realidade de questionamento das atribuições profissionais não se restringe apenas ao município de Florianópolis. Na ocasião, agentes comunitários de saúde revelaram já ter vivenciado crise de identidade profissional, uma vez que, muitas vezes, esses trabalhadores realizam atividades que não fazem parte de suas atribuições por conta da escassez de outros profissionais; com isso, sentem-se sobrecarregados e veem seu tempo disponível diminuir para a realização de suas tarefas $^{19}$. Outro estudo realizado por Almeida et al. ${ }^{20}$, em Minas Gerais, mostrou que a problemática acerca dos desvios de atividades e da dificuldade de comunicação com a equipe parece ser algo que ocorre em várias partes do território nacional, tendo essas respostas aparecido nos questionamentos diretos aos ACS, inclusive como motivo para a sensação de sobrecarga de trabalho.

Outro fator a ser considerado nessa diferença de informações (ainda que não tenha sido dito pelos ACS) é que, segundo os últimos dados disponíveis para a população da área de abrangência da UBS estudada - a partir do censo do Instituto Brasileiro de Geografia e Estatística (IBGE), de 2010, com projeção de renda e idade para 2012 -, a maior parte da população se encontra em idade economicamente ativa e com rendimento mensal entre 1/2 e 3 salários mínimos (tabela 2) 21. Desta forma, supõe-se que essa população tenha trabalho formal (ou pelos menos regular) e em período integral, o que dificulta o trabalho dos ACS, a consequente vigilância do território e também prejudica a disponibilização de números atuais nos SIS. 
Tabela 2. População da área da UBS em estudo com idade maior ou igual a 10 anos, segundo rendimento mensal em salários mínimos, ano 2012

\begin{tabular}{lrrrrr}
\hline Microárea & Até $1 / 2$ & De $1 / 2$ a 1 & De 1 a 2 & De 2 a 3 & De 3 a 5 \\
\hline 44001 & 2 & 77 & 93 & 39 & 21 \\
44002 & 5 & 48 & 143 & 56 & 43 \\
44003 & 7 & 55 & 115 & 35 & 25 \\
44004 & 6 & 100 & 208 & 110 & 87 \\
44005 & 4 & 61 & 128 & 68 & 53 \\
44006 & 4 & 40 & 119 & 46 & 36 \\
Total & $\mathbf{2 8}$ & $\mathbf{3 8 1}$ & $\mathbf{8 0 6}$ & $\mathbf{3 5 4}$ & $\mathbf{2 6 5}$ \\
\hline
\end{tabular}

Fonte: IBGE, Florianópolis, $2012 \mathbf{1 1}$ (projeção a partir do censo de 2010).

Estudos internacionais mostram que a vigilância em saúde é uma atividade importante, com melhores resultados de saúde devido à atuação do ACS. No Quênia, estudo avaliou as mortes neonatais e concluiu que, após treinamento de ACS para prestar esse atendimento, identificaram-se reduções na mortalidade. O ACS avalia os recém-nascidos para detectar sinais de doença grave durante a primeira semana de vida e referência as crianças doentes para uma unidade de saúde. Ainda segundo o estudo citado, no Reino Unido, há contribuição potencial de ACS para melhorar os resultados de saúde na atenção primária22. Logo, a atuação do ACS se faz importante para o desenvolvimento do indivíduo desde o seu nascimento, propiciando que a equipe tenha acesso aos casos que demandam mais atenção para acolher e acompanhar da melhor maneira. No entanto, a forma de atuação desse profissional deve considerar o meio no qual ele está inserido e a capacidade de atendimento das equipes de saúde do território, sendo difícil entender que uma fórmula única de atuação para todo o País seja o mais adequado - ainda que com uma linha condutora geral -, uma vez que as realidades locais são muito variadas ${ }^{\mathbf{2 3}}$.

A partir da vigilância do território realizada pelos ACS, a equipe toma consciência da sua população adscrita, facilitando as abordagens e o planejamento das atividades e rotinas da unidade, além de realizar a educação em saúde da população e de poder agir de maneira preventiva aos problemas. Considera-se, ainda, que o processo de trabalho dos ACS se torna mais prazeroso para esses profissionais quando eles observam o progresso da qualidade do atendimento à população. No entanto, nos parece que uma das questões para que isso aconteça é que os instrumentos de trabalho desse profissional, aqui com foco nos sistemas de informação, possam ser devidamente atualizados e integrados, possibilitando que as observações feitas pelos ACS sejam mais facilmente comunicadas ao restante da equipe, evitando que o preenchimento de um sistema seja feito por mera formalidade. A integração e a efetiva troca de informações entre o ACS e o restante da equipe oferece, além de melhores intervenções para a saúde da população, maior motivação e percepção de qualidade do processo de trabalho desse profissional ${ }^{5,24}$.

Sabendo de tal importância, deve-se atentar para que os ACS não sejam excluídos da centralidade dos programas de capacitação e educação em saúde, para melhor desenvolvimento das suas habilidades e potencialidades para com a comunidade e a $\mathrm{ESF}^{25}$. 


\section{Conclusões}

Por meio deste estudo, buscou-se compreender os motivos que tornaram evidente a diferença de dados nos sistemas de informação pesquisados a partir da fala e da escrita dos agentes comunitários de saúde, propondo ferramentas para enfrentamento dessa problemática em conjunto com a equipe de saúde. Logo, identificou-se que a existência de duas bases diferentes prejudica a vigilância desses casos, evidenciando que os profissionais médicos, enfermeiros e odontólogos possuem mais informações da área que os ACS.

A falta de profissionais técnicos administrativos leva à redistribuição das atividades e faz com que os ACS não fiquem dedicados em tempo integral à vigilância do território. Mas seria essa a principal justificativa para tanta diferença nos dados encontrados? Infere-se que não, já que os agentes trabalham nas atividades internas da UBS em regime de escala, mas em menor proporção de tempo - 1 para 5 -, e as diferenças entre os sistemas são significativas para serem explicadas apenas por isso.

Cabe lembrar que esses profissionais realizam treinamento antes de iniciar seu trabalho na UBS e que a equipe busca realizar capacitações junto aos ACS de maneira continuada, mesmo cientes de que devem acontecer com mais frequência. Ainda, participam das reuniões de equipe semanais e mensais da UBS, estando sempre em contato com as demandas da EqSF. Com isso, não é possível justificar essa discrepância de dados pela ausência da equipe.

Cabe aos enfermeiros, enquanto supervisores dos ACS nas equipes, observar a execução das atividades desenvolvidas pelos agentes comunitários de sua respectiva área e, junto aos demais profissionais médicos e cirurgiões-dentistas, contribuir para a identificação dos usuários que não comparecem às consultas ou que merecem mais atenção da equipe (situações prioritárias).

Sabendo que os ACS parecem estar cientes da importância do seu trabalho - identificado na aplicação dos questionários e discussões - e que há tempo suficiente para a sua realização, como deve proceder a equipe para minimizar essa diferença de dados e melhorar o planejamento de ações? O desenvolvimento do trabalho sugeriu como atividade possível que a EqSF auxilie mais os ACS durante as reuniões semanais na conferência da lista dos indivíduos em comparação com aqueles acompanhados na UBS, ou, ainda, que ajude na atualização do sistema CadFam dos dados coletados na área de abrangência, entendendo que tal atualização confere mais qualidade aos cuidados prestados à comunidade, principalmente para as pessoas que mais precisam de cuidado continuado.

Percebe-se que os ACS têm dificuldade de entender os motivos das diferenças entre os dados, limitando-se a tentar defender-se, na maior parte do tempo, e justificando que todas as pessoas são acompanhadas, ainda que relatem a carga horária administrativa como um dificultador. O restante da equipe, por outro lado, não se manifesta na concordância ou discordância dessa explicação. Mas fica evidente a todos, incluindo os pesquisadores, que a desintegração das bases de dados dificulta imensamente a integração do trabalho dessas duas partes, fato que poderia ser resolvido com a adoção de sistemas mais adequados ao processo de vigilância do território e da ESF como um todo. No entanto, tal medida não compete à EqSF, ficando fora das intervenções possíveis no escopo deste trabalho.

\section{Colaboradores}

Todos os autores do manuscrito contribuíram substancialmente com a concepção e planejamento do trabalho, análise e interpretação dos dados, revisão crítica do conteúdo e aprovação da versão final do manuscrito. 


\section{Referências}

1. Tomaz J. O agente comunitário de saúde não deve ser um 'super-herói'. Comunic. Saúde Educ. 2002; 6(10):75-94

2. Brasil. Ministério da Saúde. Política Nacional de Atenção Básica. Brasília, DF: Ministério da Saúde; 2012.

3. Brasil. Ministério da Saúde. Portaria n ${ }^{\circ}$ 2.488, de 21 de outubro de 2011. Aprova a Política Nacional de Atenção Básica. Diário Oficial da União. 22 Out 2011.

4. Escorel S, Giovanella L, Mendonça M, et al. O Programa de Saúde da Família e a construção de um novo modelo de atenção básica no Brasil. Rev. Panam Salud Publica. 2007; 21(2):164-176.

5. Fernandes J, Carrapato L, Rose E, et al. Percepções dos profissionais de saúde da atenção primária sobre qualidade no processo de trabalho. Saúde Soc. 2018; 27(2):518-530.

6. Araújo MRN, Assunção RS. A atuação do agente comunitário de saúde na promoção da saúde e na prevenção de doenças. Rev. Bras Enferm. 2014; 57(1):1925 .

7. Starfield B, Shi L, Macinko J. Contribution of Primary Care to Health Systems and Health. The Milbank Quarterly. 2005; 83(3):457-502.

8. Oliveira C, Casanova A. Vigilância da Saúde no Espaço de Práticas da Atenção Básica. Ciênc. Saúde Colet. 2009; 14(3):929-936.

9. Trentini M, Paim L. Pesquisa Covnergente Assistencial. 2. ed. Florianópolis: Insular; 2004.

10. Santos KT, Saliba NA, Moimaz SAS, et al. Agente comunitário de saúde: perfil adequado a realidade do Programa Saúde da Família? Ciênc. Saúde Colet. 2011; 16(supl1):1023-1028.

11. Brasil. Ministério da Saúde. Guia prático do agente comunitário de saúde. Brasília, DF: Ministério da
Saúde; 2009.

12. Ellen N, Silva M, Leonette D. Atividades dos Agentes Comunitários de Saúde no âmbito da Estratégia Saúde da Família : revisão integrativa da literatura. Saúde transform. soc. 2014; (84):16-21.

13. Alonso CMC, Béguin PD, Duarte FJDCM. Work of community health agents in the Family Health Strategy: meta-synthesis. Rev. Saúde Pública. 2018; 52:14.

14. Leão e Silva LO, Dias CA, Rodrigues SM, et al. Hipertensão Arterial Sistêmica: Representações Sociais de idosos sobre a doença e seu tratamento. Cad. Saúde Coletiva. 2013; 21(2):121-128.

15. Pierin AMG, Silva SSBE, Colósimo FC, et al. Chronic and asymptomatic diseases influence the control of hypertension treatment in primary care. Rev. da Esc. Enferm. 2016; 50(5):763-770.

16. Cardoso AS, Nascimento MC. Comunicação no Programa Saúde da Família : o agente de saúde como elo integrador entre a equipe e a comunidade. Ciênc. Saúde Colet. 2010; 15(supl1):1509-1520.

17. Peres CRFB, Junior ALC, Silva RF, et al. O Agente Comunitário de Saúde frente ao processo de trabalho em equipe: Facilidades e dificuldades. Rev. da Esc. Enferm. 2011; 45(4):905-911.

18. Brasil. Carteira de Serviços da Atenção Primária de Florianópolis. [internet]. Prefeitura Florianópolis. [acesso em 2018 nov 19]; 2014. Disponível em: https://drive.google.com/file/ d/o BxvFvjfrIbyweVlIYUtpMWRZZHM/ edit?usp=sharing.

19. Peserico A, Colomé J, Speroni K, et al. Atuação dos agentes comunitários de saúde na estratégia de saúde da família: percepções dos trabalhadores. Rev. enferm UFSM. 2014; 4(3):488-497.

20. Cristina M, Almeida S, Campos P, et al. Cargas de 
trabalho e processo de desgaste em Agentes Comunitários de Saúde. Rev. Esc. Enferm USP. 2016; 5050(11):95-103.

21. Brasil. Prefeitura Municipal de Florianópolis. População 2012 por rendimento e raça [internet]. [acesso em 2018 nov 19] 2012. Disponível em: http://www. pmf.sc.gov.br/sistemas/saude/unidades_saude/ populacao/2012r/uls_2012_index.php.

22. Costa S, Araújo F, Martins L, et al. Agente Comunitário de Saúde: elemento nuclear das ações em saúde. Ciênc. Saúde Colet. 2013; 18(7):2147-2156.

23. Souza MF. O agente comunitário de saúde: Uma análise dos artigos produzidos nas regiões Sul e Nordeste do Brasil [monografia] [internet]. Florianópolis: Universidade Federal de Santa Catarina; 2009. 82 p. [acesso em 2018 nov 19]. Disponível em: https://repositorio.ufsc.br/xmlui/handle/123456789/126442.

24. Cordeiro L, Soares CB. Processo de trabalho na Atenção Primária em Saúde: pesquisa-ação com Agentes Comunitários de Saúde. Ciênc. Saúde Colet. 2015; 20(11):3581-3588.

25. Pinto AAM, Fracolli LA. O trabalho do agente comunitário de saúde na perspectiva da promoção da saúde: considerações práticas. Rev. Eletrônica Enferm. 2010; 12(4):766-769.

Recebido em 27/07/2018

Aprovado em 07/10/2018

Conflito de interesses: não houve

Suporte Financeiro: inexistente 